\title{
New Results for Periodic Solution of High-Order BAM Neural Networks with Continuously Distributed Delays and Impulses
}

\author{
Chang-Bo Yang, ${ }^{1,2}$ Ting-Zhu Huang, ${ }^{1}$ and Jin-Liang Shao ${ }^{1}$ \\ ${ }^{1}$ School of Mathematical Sciences, University of Electronic Science and Technology of China, Chengdu, Sichuan 611731, China \\ ${ }^{2}$ Institute of Nonlinear Analysis, Kunming University, Kunming, Yunnan 650214, China
}

Correspondence should be addressed to Ting-Zhu Huang; tingzhuhuang@126.com

Received 12 November 2012; Accepted 24 March 2013

Academic Editor: Shiping Lu

Copyright (c) 2013 Chang-Bo Yang et al. This is an open access article distributed under the Creative Commons Attribution License, which permits unrestricted use, distribution, and reproduction in any medium, provided the original work is properly cited.

By $M$-matrix theory, inequality techniques, and Lyapunov functional method, certain sufficient conditions are obtained to ensure the existence, uniqueness, and global exponential stability of periodic solution for a new type of high-order BAM neural networks with continuously distributed delays and impulses. These novel conditions extend and improve some previously known results in the literature. Finally, an illustrative example and its numerical simulation are given to show the feasibility and correctness of the derived criteria.

\section{Introduction}

As is well known, during the hardware implementation of neural networks, time delays are inevitable due to finite switching speeds of the amplifiers and communication time, which may bring about complex influence on the system such as oscillation and instability $[1,2]$. On the other hand, impulsive effects wildly exist in many realistic networks [3, 4], which may be caused by witching phenomenon, sudden changes, or other unexpected noise. Therefore, it is more appropriate to consider delay and impulsive effects when modeling neural networks, and many researches on various kinds of neural networks with delays, impulses, or both of them have been available [5-12]. (See Figures 1(a), 1(b), and $1(\mathrm{c}))$.

Bidirectional associative memory (BAM) neural networks, as an extension of the unidirectional autoassociator of Hofield neural network [13], was firstly introduced by Kosko [14]. Due to its wide application in pattern recognition, associative memory, image, and signal processing, BAM neural networks with delays and impulses have been extensively studied in the past few decades [15-22]. In addition, it is worth noting that high-order neural networks structures have advantages of stronger storage capacity, faster convergence rate, and higher fault tolerance, and these merits have been successfully used in pattern recognition [23]. Thus, it is important to investigate BAM neural networks with high-order terms, which is called high-order BAM neural networks.

In this paper, we will consider a new type of highorder BAM neural networks with continuously distributed delays and impulses, which can be described by the following integrodifferential equations:

$$
\begin{aligned}
& \frac{d x_{i}(t)}{d t} \\
& =-a_{i}(t) x_{i}(t)+\sum_{j=1}^{m} c_{i j}(t) \int_{0}^{\infty} K_{i j}(s) f_{j}\left(y_{j}(t-s)\right) d s \\
& \quad+\sum_{j=1}^{m} \sum_{q=1}^{m} s_{i j q}(t) \int_{0}^{\infty} R_{i j q}(s) f_{j}\left(y_{j}(t-s)\right) f_{q}\left(y_{q}(t-s)\right) d s \\
& \quad+I_{i}(t), \quad t \neq t_{k}, \\
& x_{i}\left(t_{k}^{+}\right)=x_{i}\left(t_{k}^{-}\right)+\Delta x_{i}\left(t_{k}\right)=H_{i k}\left(x_{i}\left(t_{k}^{-}\right)\right), \\
& \quad i=1,2, \ldots, n, \quad k \in \mathbb{Z} \triangleq\{1,2, \ldots\},
\end{aligned}
$$




$$
\begin{gathered}
\frac{d y_{j}(t)}{d t}=-b_{j}(t) y_{j}(t)+\sum_{i=1}^{n} d_{j i}(t) \int_{0}^{\infty} \widetilde{K}_{j i}(s) g_{i}\left(x_{i}(t-s)\right) d s \\
+\sum_{i=1}^{n} \sum_{p=1}^{n} e_{j i p}(t) \int_{0}^{\infty} \widetilde{R}_{j i p}(s) g_{i}\left(x_{i}(t-s)\right) g_{p} \\
\times\left(x_{p}(t-s)\right) d s+J_{j}(t), \quad t \neq t_{k}, \\
y_{j}\left(t_{k}^{+}\right)=y_{j}\left(t_{k}^{-}\right)+\Delta y_{j}\left(t_{k}\right)=E_{j k}\left(y_{j}\left(t_{k}^{-}\right)\right), \\
j=1,2, \ldots, m, k \in \mathbb{Z} \triangleq\{1,2, \ldots\},
\end{gathered}
$$

where $\Delta x_{i}\left(t_{k}\right)$ and $\Delta y_{j}\left(t_{k}\right)$ are the impulses at moments $t_{k}$ and $t_{1}<t_{2}<\cdots$ is a strictly increasing sequence such that $\lim _{k \rightarrow \infty} t_{k}=\infty$. And $x_{i}(t)$ and $y_{j}(t)$ are the activations of the $i$ th neuron and the $j$ th neuron, respectively; $a_{i}(t)>0$ and $b_{j}(t)>0$ denote the passive decay rates; $c_{i j}(t), d_{j i}(t), s_{i j q}(t), e_{j i p}(t)$ are the first- and second-order connection weights of the neural networks, respectively; $I_{i}(t)$ and $J_{j}(t)$ are the external inputs.

Clearly, system (1) is a more general form of BAM neural networks, which has been widely applied in areas of science and engineering [24], such as neurobiology, image classification, and image recognition. In recent years, studies of such kind of neural networks with delays and impulses have received considerable interest, and some results have been reported in [25-30]. In particular, authors in [2528] have discussed the stability of equilibrium point for a kind of impulsive high-order BAM neural networks with discrete delays by different methods, such as linear matrix inequality (LMI), Razumikhin technique. Subsequently, Huo et al. [29] and Yang [30] studied the existence of periodic solution and its exponential stability for an impulsive highorder BAM neural network with discrete delays by using the theory of coincidence degree and Lyapunov functional method. However, to the best of our knowledge, there are few results on the existence, uniqueness, and global exponential stability of periodic solution for system (1) with continuously distributed delays.

The main propose of this paper is to study the periodicity of system (1) with distributed delays and general impulsive effects. It should be noticed that some new criteria on the existence and uniqueness of periodic solution for system (1) are established by combining the general $\|\cdot\|_{r}$ (see Notations) and analytical techniques, which is different from the conventional continuation theorem of coincidence degree theory used in $[29,30]$. In addition, it is worth mentioning that the impulsive part in this paper is not necessarily bounded and linear, which makes its applications more extensive.

The rest of this paper is organized as follows. In Section 2, some assumptions, definitions, and important lemmas are given. In Section 3, the main results and some remarks are presented. In Section 4, an example and its numerical simulation are provided. Finally, some conclusions are summarized in Section 5.

\section{Preliminaries}

Notations. Throughout this paper, $\mathfrak{R}$ and $\mathfrak{R}^{n}$ denote the set of real numbers and $n$-dimensional vector space, respectively. The symbol $(\cdot)^{T}$ denotes the transpose of a vector or a matrix. Take $\|x\|_{r}=\left(\sum_{i=1}^{n}\left|x_{i}\right|^{r}\right)^{1 / r}$ with integer $r \in[1, \infty)$. Clearly, $\|\cdot\|_{1},\|\cdot\|_{2}$ are special cases of $\|\cdot\|_{r}$ with $r=1,2$, respectively, which are used to investigate the dynamics of various kinds of neural networks in $[6-8,10,12,15-17,19,21,22,25-31]$. Denote $C^{*} \triangleq\left\{\phi:(-\infty, 0] \rightarrow \mathfrak{R}^{n+m} \mid \phi(s)\right.$ is continuous for all but at most countable points $s \in(-\infty, 0]$ and at these points $s \in(-\infty, 0], \phi\left(s^{+}\right)$and $\phi\left(s^{-}\right)$exist, $\left.\phi(s)=\phi\left(s^{-}\right) \cdot\right\}$ and define the norm $\|\phi\|_{\Delta}$ by

$$
\|\phi\|_{\Delta}=\left(\sum_{i=1}^{n} \sup _{s \in(-\infty, 0]}\left|\phi_{x_{i}}(s)\right|^{r}+\sum_{j=1}^{m} \sup _{s \in(-\infty, 0]}\left|\phi_{y_{j}}(s)\right|^{r}\right)^{1 / r},
$$

where $\phi=\left(\phi_{x}^{T}, \phi_{y}^{T}\right)^{T}=\left(\phi_{x_{1}}, \phi_{x_{2}}, \ldots, \phi_{x_{n}}, \phi_{y_{1}}, \phi_{y_{2}}, \ldots, \phi_{y_{m}}\right)^{T}$, and then $C^{*}$ is a Banach space with topology of the uniform convergence. In addition, system (1) is supplemented with initial values

$$
\begin{gathered}
x_{i}(s)=\phi_{x_{i}}(s), \quad s \in(-\infty, 0], i=1,2, \ldots, n, \\
y_{j}(s)=\phi_{y_{j}}(s), \quad s \in(-\infty, 0], j=1,2, \ldots, m .
\end{gathered}
$$

As usual, we have the following assumptions for system (1).

$\left(\mathrm{S}_{1}\right)$ Functions $a_{i}(t), a_{j}(t), c_{i j}(t), d_{j i}(t), s_{i j q}(t), e_{j i p}(t)$, $I_{i}(t)$, and $J_{j}(t)$ are $\omega$-periodic and bounded on $[0,+\infty)$ such that $a_{i}(t) \geq a_{i}^{-}>0, b_{j}(t) \geq b_{j}^{-}>0,\left|c_{i j}(t)\right| \leq c_{i j}^{+},\left|d_{j i}(t)\right| \leq$ $d_{j i}^{+},\left|s_{i j q}(t)\right| \leq s_{i j q}^{+},\left|e_{j i p}(t)\right| \leq e_{j i p}^{+},\left|I_{i}(t)\right| \leq I_{i},\left|J_{j}(t)\right| \leq J_{j}$ for $i, p=1,2, \ldots, n, j, q=1,2, \ldots, m$.

$\left(\mathrm{S}_{2}\right)$ The activation functions $g_{i}(\cdot), g_{p}(\cdot), f_{j}(\cdot)$, and $f_{q}(\cdot)$ are bounded and Lipschitz continuous on $\mathfrak{R}$; that is, there exist positive numbers $M_{j}^{f}, M_{q}^{f}, M_{i}^{g}, M_{p}^{g}$ and $L_{i}^{g}, L_{j}^{f}$ such that

$$
\begin{aligned}
& \left|f_{j}(x)\right| \leq M_{j}^{f}, \quad\left|f_{q}(x)\right| \leq M_{q}^{f}, \\
& \left|f_{j}(x)-f_{j}(y)\right| \leq L_{j}^{f}|x-y|, \\
& \left|g_{i}(x)\right| \leq M_{i}^{g}, \quad\left|g_{p}(x)\right| \leq M_{p}^{g}, \\
& \left|g_{i}(x)-g_{i}(y)\right| \leq L_{i}^{g}|x-y|,
\end{aligned}
$$

for $i, p=1,2, \ldots, n, j, q=1,2, \ldots, m$ and $x, y \in \Re$.

$\left(\mathrm{S}_{3}\right)$ The delay kernel functions $K_{i j}(\cdot), \widetilde{K}_{j i}(\cdot), R_{i j q}(\cdot)$, and $\widetilde{R}_{i j p}(\cdot)$ are piecewise continuous functions from $[0, \infty)$ to $[0, \infty)$ and satisfy $K_{i j}(s) \leq \mathscr{K}(s), \widetilde{K}_{j i}(s) \leq \mathscr{K}(s), R_{i j q}(\cdot) \leq$ $\mathscr{R}(s)$, and $\widetilde{R}_{i j p}(s) \leq \mathscr{R}(s)$ for $s \in[0, \infty), i, p=$ $1,2, \ldots, n, j, q=1,2, \ldots, m$, where $\mathscr{K}(s), \mathscr{R}(s)$ satisfy

$$
\begin{aligned}
& \int_{0}^{\infty} \mathscr{K}(s) d s=1, \quad \int_{0}^{\infty} e^{\varepsilon s} \mathscr{K}(s) d s<+\infty, \\
& \int_{0}^{\infty} \mathscr{R}(s) d s=1, \quad \int_{0}^{\infty} e^{\varepsilon s} \mathscr{R}(s) d s<+\infty,
\end{aligned}
$$




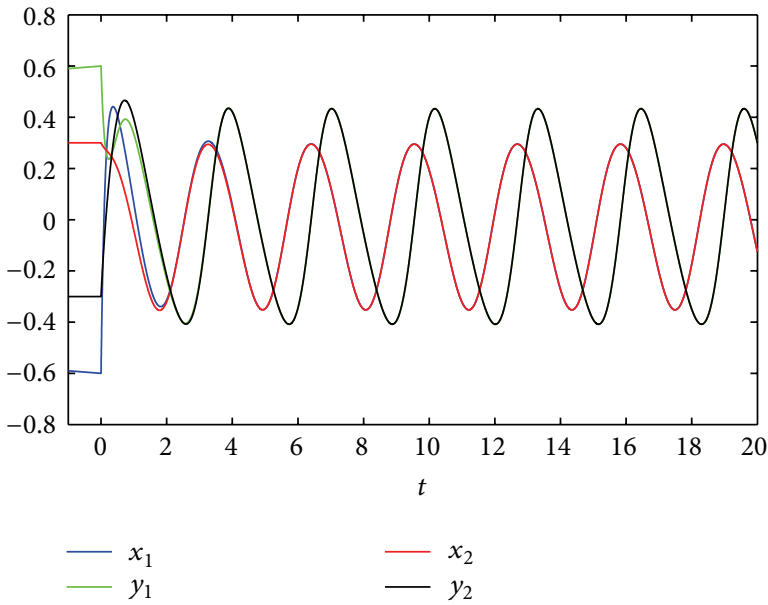

(a) State response of the impulse-free system (51)

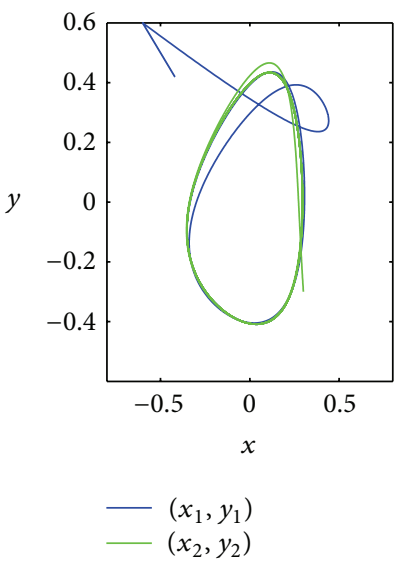

(c) Phase plots of system (51) without and with impulses, respectively

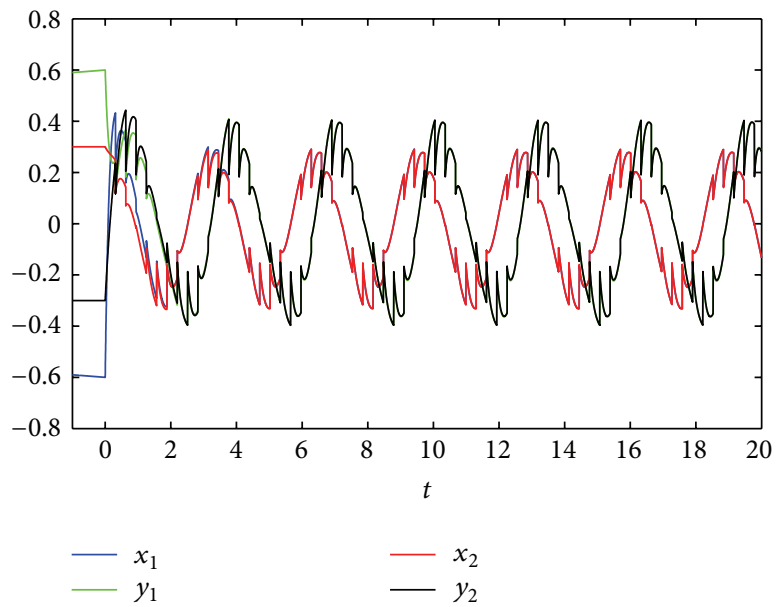

(b) State response of the impulsive system (51)

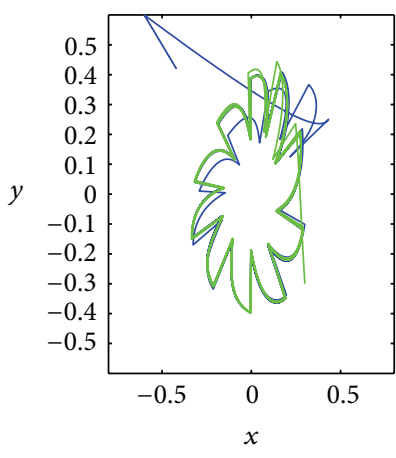

$-\left(x_{1}, y_{1}\right)$

$-\left(x_{2}, y_{2}\right)$

FIGURE 1: It is confirmed that the system (51) has a unique $\pi$-periodic solution. Here $\left(x_{1}, y_{1}\right)$ and $\left(x_{2}, y_{2}\right)$ denote two pairs of solution of system (51) with different initial conditions $\phi_{1}(s)=\left(-e^{0.01 s}+0.4, e^{0.01 s}-0.4\right)^{T}$ and $\phi_{2}(s)=(0.3,-0.3)^{T}$ for $s \in[-1,0]$ and $\gamma=0.5, t_{k}-t_{k-1}=$ $\pi, k=1,2, \ldots$

in which $\varepsilon$ denotes some positive constant number. For more information on these delay kernels, one can refer to $[5,9,15$, $18,22]$.

$\left(\mathrm{S}_{4}\right) \Omega$ is an $M$-matrix, where $r \geq 1$,

$$
\Omega=\left(\begin{array}{cc}
r A-(r-1) G & -L^{f} \Pi \\
-L^{g} \Gamma & r B-(r-1) F
\end{array}\right)
$$

$A=\operatorname{diag}\left(a_{1}^{-}, a_{2}^{-}, \ldots, a_{n}^{-}\right), \quad B=\operatorname{diag}\left(b_{1}^{-}, b_{2}^{-}, \ldots, b_{m}^{-}\right)$,

$G=\operatorname{diag}\left(G_{1}, G_{2}, \ldots, G_{n}\right), \quad F=\operatorname{diag}\left(F_{1}, F_{2}, \ldots, F_{m}\right)$,

$L^{g}=\operatorname{diag}\left(L_{1}^{g}, L_{2}^{g}, \ldots, L_{n}^{g}\right), \quad L^{f}=\operatorname{diag}\left(L_{1}^{f}, L_{2}^{f}, \ldots, L_{m}^{f}\right)$,

$G_{i}=\sum_{j=1}^{m}\left[c_{i j}^{+}+\sum_{q=1}^{m}\left(s_{i j q}^{+}+s_{i q j}^{+}\right) M_{q}^{f}\right] L_{j}^{f}$,

$F_{j}=\sum_{i=1}^{n}\left[d_{j i}^{+}+\sum_{p=1}^{n}\left(e_{j i p}^{+}+e_{j p i}^{+}\right) M_{p}^{g}\right] L_{i}^{g}$,

$$
\Pi=\left(\Pi_{i j}\right)_{n \times m}=c_{i j}^{+}+\sum_{q=1}^{m}\left(s_{i j q}^{+}+s_{i q j}^{+}\right) M_{q}^{f},
$$$$
\Gamma=\left(\Gamma_{j i}\right)_{m \times n}=d_{j i}^{+}+\sum_{p=1}^{n}\left(e_{j i p}^{+}+e_{j p i}^{+}\right) M_{p}^{g} .
$$

Definition 1. A function $z(t, \phi)=\left(x_{1}(t, \phi), x_{2}(t, \phi), \ldots\right.$, $\left.x_{n}(t, \phi), y_{1}(t, \phi), y_{2}(t, \phi), \ldots, y_{m}(t, \phi)\right)^{T} \in \mathfrak{R}^{n+m}$ is said to be the solution of system (1) with initial condition $\phi \in C^{*}$ if the following two conditions are satisfied.

(1) $z(t, \phi)$ is piecewise continuous with first kind discontinuity at the points $t_{k}, k \in \mathbb{Z}$. Moreover, $z(t, \phi)$ is left continuous at each of the discontinuity points.

(2) $z(t, \phi)$ satisfies system (1) for $t \geq 0$ and $z(s)=\phi(s)$ for $s \in(-\infty, 0]$.

Definition 2. The periodic solution $z^{*}\left(t, \phi^{*}\right)$ of system (1) is said to be globally exponentially stable, if there exist constants 
$\mathscr{M} \geq 1$ and $\alpha>0$ such that any other solution $z(t, \phi)$ of system (1) satisfies

$$
\left\|z(t, \phi)-z^{*}\left(t, \phi^{*}\right)\right\|_{r} \leq M e^{-\alpha t}\left\|\phi-\phi^{*}\right\|_{\Delta}, \quad t \geq 0 .
$$

Lemma 3 (see [32]). Let $A \in \mathbb{Z}^{n \times n}$, where $\mathbb{Z}^{n \times n}$ is a set of $n \times n$ matrices with nonpositive off-diagonal elements. $A$ is an M-matrix if and only if there exists a positive vector $\lambda=$ $\left(\lambda_{1}, \lambda_{2}, \ldots, \lambda_{n}\right)^{T}$ such that $\sum_{j=1}^{n} a_{j i} \lambda_{j}>0$ or $\sum_{j=1}^{n} a_{i j} \lambda_{j}>0$.

Lemma 4. Assume that assumptions $\left(S_{3}\right)$ and $\left(S_{4}\right)$ hold; then there exist positive constants $\varepsilon$ and $\lambda_{1}, \lambda_{2}, \ldots, \lambda_{n+m}$ such that

$$
\begin{aligned}
& P_{i}(\varepsilon)= {\left[-\varepsilon+r a_{i}^{-}-(r-1) G_{i}\right] \lambda_{i} } \\
&-\sum_{j=1}^{m} \lambda_{n+j} d_{j i}^{+} L_{i}^{g} \int_{0}^{\infty} \mathscr{K}(s) e^{\varepsilon s} d s \\
&-\sum_{j=1}^{m} \sum_{p=1}^{n} \lambda_{n+j}\left(e_{j i p}^{+}+e_{j p i}^{+}\right) M_{p}^{g} L_{i}^{g} \\
& \times \int_{0}^{\infty} \mathscr{R}(s) e^{\varepsilon s} d s \geq 0, \\
& Q_{j}(\varepsilon)=\left.-\varepsilon+r b_{j}^{-}-(r-1) F_{j}\right) \lambda_{n+j} \\
&-\sum_{i=1}^{n} \lambda_{i} c_{i j}^{+} L_{j}^{f} \int_{0}^{\infty} \mathscr{K}(s) e^{\varepsilon s} d s \\
&-\sum_{i=1}^{n} \sum_{q=1}^{m} \lambda_{i}\left(s_{i j q}^{+}+s_{i q j}^{+}\right) M_{q}^{f} L_{j}^{f} \\
& \times \int_{0}^{\infty} \mathscr{R}(s) e^{\varepsilon s} d s \geq 0,
\end{aligned}
$$

for $i, p=1,2, \ldots, n, j, q=1,2, \ldots, m$.

Proof. Construct the aided functions as follows:

$$
\begin{aligned}
P_{i}\left(\vartheta_{i}\right)= & {\left[-\vartheta_{i}+r a_{i}^{-}-(r-1) G_{i}\right] \lambda_{i} } \\
& -\sum_{j=1}^{m} \lambda_{n+j} d_{j i}^{+} L_{i}^{g} \int_{0}^{\infty} \mathscr{K}(s) e^{\vartheta_{i} s} d s \\
& -\sum_{j=1}^{m} \sum_{p=1}^{n} \lambda_{n+j}\left(e_{j i p}^{+}+e_{j p i}^{+}\right) M_{p}^{g} L_{i}^{g} \int_{0}^{\infty} \mathscr{R}(s) e^{\vartheta_{i} s} d s, \\
Q_{j}\left(\mu_{j}\right)= & \left.-\mu_{j}+r b_{j}^{-}-(r-1) F_{j}\right) \lambda_{n+j} \\
& -\sum_{i=1}^{n} \lambda_{i} c_{i j}^{+} L_{j}^{f} \int_{0}^{\infty} \mathscr{K}(s) e^{\mu_{j} s} d s \\
& -\sum_{i=1}^{n} \sum_{q=1}^{m} \lambda_{i}\left(s_{i j q}^{+}+s_{i q j}^{+}\right) M_{q}^{f} L_{j}^{f} \int_{0}^{\infty} \mathscr{R}(s) e^{\mu_{j} s} d s
\end{aligned}
$$

for $i, p=1,2, \ldots, n, j, q=1,2, \ldots, m$. Using Lemma 3 and assumptions $\left(\left(\mathrm{S}_{3}\right)-\left(\mathrm{S}_{4}\right)\right)$, it is easy to deduce that $(8)$ hold by similar proof in $[7,15-18]$. For concise, it is omitted here.
Lemma 5. Let the integer $r \geq 1$; then the inequality holds as follows:

$$
(n+m)^{(1-r) / r} \sum_{i=1}^{n+m}\left|z_{i}\right| \leq\left(\sum_{i=1}^{n+m}\left|z_{i}\right|^{r}\right)^{1 / r}
$$

for all $z=\left(z_{1}, z_{2} \ldots, z_{n+m}\right)^{T} \in \mathfrak{R}^{n+m}$.

Proof. Obviously, the inequality (10) with $r=1$ is trivial. When $r>1$, consider the aided function $g(x)=x^{r}, x \geq 0$. It is claimed that $g(x)$ is convex since $g^{\prime \prime}(x)=r(r-1) x^{r-1}>0$, $r>1$ for $x>0$. Let $x=(1 /(n+m)) \sum_{i=1}^{n+m}\left|z_{i}\right|$; by Jensen's inequality, we have

$$
\frac{1}{(n+m)^{r}}\left(\sum_{i=1}^{n+m}\left|z_{i}\right|\right)^{r} \leq \frac{1}{n+m} \sum_{i=1}^{n+m}\left|z_{i}\right|^{r},
$$

which implies that the inequality (10) holds. This completes the proof.

\section{Main Results}

Firstly, let $z(t, \phi)=\left(x_{1}(t, \phi), x_{2}(t, \phi), \ldots, x_{n}(t, \phi), y_{1}(t, \phi)\right.$, $\left.y_{2}(t, \phi), \ldots, y_{m}(t, \phi)\right)^{T}$ and $z(t, \varphi)=\left(x_{1}(t, \varphi), x_{2}(t, \varphi), \ldots\right.$, $\left.x_{n}(t, \varphi), y_{1}(t, \varphi), y_{2}(t, \varphi), \ldots, y_{m}(t, \varphi)\right)^{T}$ be any two solutions of system (1) through $\phi, \psi \in C^{*}$, respectively; then we have the following useful lemma.

Lemma 6. Under assumptions $\left(\left(S_{1}\right)-\left(S_{4}\right)\right)$, if the following two conditions hold:

$\left(\mathrm{S}_{5}\right) H_{i k}(\cdot), E_{j k}(\cdot)$ are Lipschitz continuous on $\mathfrak{R}$; that $i$, there exist positive constants $H_{i k}, E_{j k}$ such that $\left|H_{i k}(x)-H_{i k}(y)\right| \leq H_{i k}|x-y|,\left|E_{j k}(x)-E_{j k}(y)\right| \leq$ $E_{j k}|x-y|$ for $i=1,2, \ldots, n, j=1,2, \ldots, m, k \in \mathbb{Z}$ and $x, y \in \mathfrak{R}$;

$\left(\mathrm{S}_{6}\right)$ there exists $\theta$ such that $\ln \eta_{k}^{r} /\left(t_{k}-t_{k-1}\right) \leq \theta<\varepsilon$, where $t_{0}=0, \eta_{k}=\max \left\{1, \max _{1 \leq i \leq n} H_{i k}, \max _{1 \leq j \leq m} E_{j k}\right\}, k \in$ $\mathbb{Z}$, and the scalar $\varepsilon$ is estimated by (8).

Then, the following inequality holds:

$$
\|z(t, \phi)-z(t, \varphi)\|_{r} \leq \mathscr{M} e^{-\alpha t}\|\phi-\varphi\|_{\Delta}, \quad t \geq 0
$$

where the constants $\mathscr{M} \geq 1$ and $\alpha>0$ are to be determined later.

Proof. To be convenient, let

$$
\begin{gathered}
X_{i}(t)=\left|x_{i}(t, \phi)-x_{i}(t, \varphi)\right|, \quad i=1,2, \ldots, n, \\
Y_{j}(t)=\left|y_{j}(t, \phi)-y_{j}(t, \varphi)\right|, \quad j=1,2, \ldots, m .
\end{gathered}
$$


It follows from $\left(\left(S_{1}\right)-\left(S_{3}\right)\right)$ that

$$
\begin{aligned}
& D^{+} X_{i}(t) \\
& \leq-a_{i}^{-} X_{i}(t)+\sum_{j=1}^{m} c_{i j}^{+} L_{j}^{f} \int_{0}^{\infty} \mathscr{K}(s) Y_{j}(t-s) d s \\
& \quad+\sum_{j=1}^{m} \sum_{q=1}^{m} s_{i j q}^{+} \\
& \quad \times \int_{0}^{\infty} \mathscr{R}(s)\left(f_{j}\left(x_{j}(t-s, \phi)\right) f_{q}\left(x_{q}(t-s, \phi)\right)\right. \\
& \quad-f_{j}\left(x_{j}(t-s, \varphi)\right) f_{q}\left(x_{q}(t-s, \phi)\right) \\
& \quad+f_{j}\left(x_{j}(t-s, \varphi)\right) f_{q}\left(x_{q}(t-s, \phi)\right) \\
& \left.\quad-f_{j}\left(x_{j}(t-s, \varphi)\right) f_{q}\left(x_{q}(t-s, \varphi)\right)\right) d s \\
& \leq-a_{i}^{-} X_{i}(t)+\sum_{j=1}^{m} c_{i j}^{+} L_{j}^{f} \int_{0}^{\infty} \mathscr{K}(s) Y_{j}(t-s) d s \\
& +\sum_{j=1}^{m} \sum_{q=1}^{m}\left(s_{i j q}^{+}+s_{i q j}^{+}\right) M_{q}^{f} L_{j}^{f} \int_{0}^{\infty} \mathscr{R}(s) Y_{j}(t-s) d s,
\end{aligned}
$$

for $t \geq 0, t \neq t_{k}, k \in \mathbb{Z}, i=1,2, \ldots, n$. Similarly, we have

$$
\begin{aligned}
D^{+} Y_{j}(t) \leq & -b_{j}^{-} Y_{j}(t)+\sum_{j=1}^{m} d_{j i}^{+} L_{i}^{g} \int_{0}^{\infty} \mathscr{K}(s) X_{i}(t-s) d s \\
& +\sum_{i=1}^{n} \sum_{p=1}^{n}\left(e_{j i p}^{+}+e_{j p i}^{+}\right) M_{p}^{g} L_{i}^{g} \int_{0}^{\infty} \mathscr{R}(s) X_{i}(t-s) d s,
\end{aligned}
$$

for $t \geq 0, t \neq t_{k}, k \in \mathbb{Z}, j=1,2, \ldots, m$. Also,

$$
\begin{aligned}
X_{i}\left(t_{k}+0\right) & =\left|H_{i k}\left(x_{i}\left(t_{k}, \phi\right)\right)-H_{i k}\left(x_{i}\left(t_{k}, \varphi\right)\right)\right| \\
& \leq H_{i k} X_{i}\left(t_{k}\right), \quad k \in \mathbb{Z}, i=1,2, \ldots, n, \\
Y_{j}\left(t_{k}+0\right) & =\left|E_{j k}\left(y_{j}\left(t_{k}, \phi\right)\right)-E_{j k}\left(y_{j}\left(t_{k}, \varphi\right)\right)\right| \\
& \leq E_{j k} Y_{j}\left(t_{k}\right), \quad k \in \mathbb{Z}, j=1,2, \ldots, m .
\end{aligned}
$$

Now define

$$
\begin{gathered}
U_{i}(t)=e^{\varepsilon t}\left(X_{i}(t)\right)^{r}, \quad i=1,2, \ldots, n, \\
V_{j}(t)=e^{\varepsilon t}\left(Y_{j}(t)\right)^{r}, \quad j=1,2, \ldots, m .
\end{gathered}
$$

By using Young inequality $a^{p} b^{q} \leq p a+q b$, where $a, b, p, q>0$ and $1 / p+1 / q=1$, it follows from (14)-(15) that

$$
\begin{aligned}
& D^{+} U_{i}(t) \\
& \leq\left(\varepsilon-r a_{i}^{-}\right) U_{i}(t) \\
& +r e^{\varepsilon t} \sum_{j=1}^{m} c_{i j}^{+} L_{j}^{f} \\
& \quad \times \int_{0}^{\infty} \mathscr{K}(s)\left(\left(X_{i}(t)\right)^{r}\right)^{(r-1) / r}\left(\left(Y_{j}(t-s)\right)^{r}\right)^{1 / r} d s \\
& +r e^{\varepsilon t} \sum_{j=1}^{m} \sum_{q=1}^{m}\left(s_{i j q}^{+}+s_{i q j}^{+}\right) M_{q}^{f} L_{j}^{f} \\
& \quad \times \int_{0}^{\infty} \mathscr{R}(s)\left(\left(X_{i}(t)\right)^{r}\right)^{(r-1) / r}\left(\left(Y_{j}(t-s)\right)^{r}\right)^{1 / r} d s \\
& +\quad\left(\varepsilon-r a_{i}^{-}+(r-1) G_{i}\right) U_{i}(t) \\
& +\sum_{j=1}^{m} c_{i j}^{+} L_{j}^{f} \int_{0}^{\infty} \mathscr{K}(s) e^{\varepsilon s} V_{j}(t-s) d s \\
& +\sum_{j=1}^{m} \sum_{q=1}^{m}\left(s_{i j q}^{+}+s_{i q j}^{+}\right) M_{q}^{f} L_{j}^{f} \int_{0}^{\infty} \mathscr{R}(s) e^{\varepsilon s} V_{j}(t-s) d s,
\end{aligned}
$$

for $t \geq 0, t \neq t_{k}, k \in \mathbb{Z}, i=1,2, \ldots, n$. Similarly, we have

$$
\begin{aligned}
D^{+} V_{j} & (t) \\
\leq & \left(\varepsilon-r b_{j}^{-}+(r-1) F_{j}\right) V_{j}(t) \\
& +\sum_{i=1}^{n} d_{j i}^{+} L_{i}^{g} \int_{0}^{\infty} \mathscr{K}(s) e^{\varepsilon s} U_{i}(t-s) d s \\
& +\sum_{i=1}^{n} \sum_{p=1}^{n}\left(e_{j i p}^{+}+e_{j p i}^{+}\right) M_{p}^{g} L_{i}^{g} \int_{0}^{\infty} \mathscr{R}(s) e^{\varepsilon s} U_{i}(t-s) d s,
\end{aligned}
$$

for $t \geq 0, t \neq t_{k}, k \in \mathbb{Z}, j=1,2, \ldots, m$. Also

$$
\begin{gathered}
U_{i}\left(t_{k}+0\right) \leq H_{i k}^{r} U_{i}\left(t_{k}\right), \quad k \in \mathbb{Z}, i=1,2, \ldots, n, \\
V_{j}\left(t_{k}+0\right) \leq E_{j k}^{r} V_{j}\left(t_{k}\right), \quad k \in \mathbb{Z}, j=1,2, \ldots, m .
\end{gathered}
$$

Consider the candidate Lyapunov-Krasovskii functional as follows:

$$
\mathbb{V}(t)=\mathbb{V}_{1}(t)+\mathbb{V}_{2}(t)
$$


where

$\mathbb{V}_{1}(t)$

$$
\begin{aligned}
& =\sum_{i=1}^{n} \lambda_{i}\left[U_{i}(t)+\sum_{j=1}^{m} c_{i j}^{+} L_{j}^{f}\right. \\
& \times \int_{0}^{\infty} \mathscr{K}(s) e^{\varepsilon s}\left(\int_{t-s}^{t} V_{j}(z) d z\right) d s \\
& +\sum_{j=1}^{m} \sum_{q=1}^{m}\left(s_{i j q}^{+}+s_{i q j}^{+}\right) M_{q}^{f} L_{j}^{f} \\
& \left.\times \int_{0}^{\infty} \mathscr{R}(s) e^{\varepsilon s}\left(\int_{t-s}^{t} V_{j}(z) d z\right) d s\right], \\
& =\sum_{j=1}^{n} \lambda_{n+j}\left[V_{j}(t)+\sum_{i=1}^{n} d_{j i}^{+} L_{i}^{g}\right. \\
& \times \int_{0}^{\infty} \mathscr{K}(s) e^{\varepsilon s}\left(\int_{t-s}^{t} U_{i}(z) d z\right) d s \\
& +\sum_{i=1}^{n} \sum_{p=1}^{n}\left(e_{j i p}^{+}+e_{j p i}^{+}\right) M_{p}^{g} L_{i}^{g} \\
& \left.\times \int_{0}^{\infty} \mathscr{R}(s) e^{\varepsilon s}\left(\int_{t-s}^{t} U_{i}(z) d z\right) d s\right] .
\end{aligned}
$$

$\mathbb{V}_{2}(t)$

When $t \neq t_{k}, k \in \mathbb{Z}$, calculating the upper right Dini derivative of $\mathbb{V}_{1}(t)$ along the solutions of system (1), we get

$$
\begin{aligned}
D^{+} \mathbb{V}_{1}(t) & \\
=\sum_{i=1}^{n} \lambda_{i}\left[D^{+} U_{i}(t)\right. & \\
& +\sum_{j=1}^{m} c_{i j}^{+} L_{j}^{f} \int_{0}^{\infty} \mathscr{K}(s) e^{\varepsilon s}\left(V_{j}(t)-V_{j}(t-s)\right) d s \\
& +\sum_{j=1}^{m} \sum_{q=1}^{m}\left(s_{i j q}^{+}+s_{i q j}^{+}\right) M_{q}^{f} L_{j}^{f} \\
\leq & \times \int_{i=1}^{\infty} \lambda_{i}\left[\left(\varepsilon-r a_{i}^{-}+(r-1) G_{i}\right) U_{i}(t)\right. \\
& \left.\left.+\sum_{j=1}^{m} c_{i j}^{+} L_{j}^{f} \int_{0}^{\infty} \mathscr{K}(s) e^{\varepsilon s} d s V_{j}(t)-V_{j}(t-s)\right) d s\right]
\end{aligned}
$$

$$
+\sum_{j=1}^{m} \sum_{q=1}^{m}\left(s_{i j q}^{+}+s_{i q j}^{+}\right) M_{q}^{f} L_{j}^{f}
$$$$
\left.\times \int_{0}^{\infty} \mathscr{R}(s) e^{\varepsilon s} d s V_{j}(t)\right] .
$$

Similarly, we have

$$
\begin{aligned}
& D^{+} \mathbb{V}_{2}(t) \\
& \leq \sum_{j=1}^{n} \lambda_{n+j}\left[\left(\varepsilon-r b_{j}^{-}+(r-1) F_{j}\right) V_{j}(t)\right. \\
& +\sum_{i=1}^{n} d_{j i}^{+} L_{i}^{g} \int_{0}^{\infty} \mathscr{K}(s) e^{\varepsilon s} d s U_{i}(t) \\
& +\sum_{i=1}^{n} \sum_{p=1}^{n}\left(e_{j i p}^{+}+e_{j p i}^{+}\right) M_{p}^{g} L_{i}^{g} \\
& \left.\quad \times \int_{0}^{\infty} \mathscr{R}(s) e^{\varepsilon s} d s U_{i}(t)\right] .
\end{aligned}
$$

Therefore, by Lemma 4, we obtain that

$$
\begin{aligned}
D^{+} \mathbb{V}(t) & \leq D^{+} \mathbb{V}_{1}(t)+D^{+} \mathbb{V}_{2}(t) \\
& \leq-\sum_{i=1}^{n} P_{i}(\varepsilon) U_{i}(t)-\sum_{j=1}^{m} Q_{j}(\varepsilon) V_{j}(t) \\
& \leq 0
\end{aligned}
$$

When $t=t_{k}, k \in \mathbb{Z}$, we have

$$
\begin{aligned}
& \mathbb{V}\left(t_{k}+0\right) \\
& =\sum_{i=1}^{n} \lambda_{i}\left[U_{i}\left(t_{k}+0\right)\right. \\
& +\sum_{j=1}^{m} c_{i j}^{+} L_{j}^{f} \int_{0}^{\infty} \mathscr{K}(s) e^{\varepsilon s}\left(\int_{t_{k}+0-s}^{t_{k}+0} V_{j}(z) d z\right) d s \\
& +\sum_{j=1}^{m} \sum_{q=1}^{m}\left(s_{i j q}^{+}+s_{i q j}^{+}\right) M_{q}^{f} L_{j}^{f} \\
& \left.\times \int_{0}^{\infty} \mathscr{R}(s) e^{\varepsilon s}\left(\int_{t_{k}+0-s}^{t_{k}+0} V_{j}(z) d z\right) d s\right]
\end{aligned}
$$




$$
\begin{aligned}
& \sum_{j=1}^{n} \lambda_{n+j}\left[V_{j}\left(t_{k}+0\right)\right. \\
& +\sum_{i=1}^{n} d_{j i}^{+} L_{i}^{g} \int_{0}^{\infty} \mathscr{K}(s) e^{\varepsilon s}\left(\int_{t_{k}+0-s}^{t_{k}+0} U_{i}(z) d z\right) d s \\
& +\sum_{i=1}^{n} \sum_{p=1}^{n}\left(e_{j i p}^{+}+e_{j p i}^{+}\right) M_{p}^{g} L_{i}^{g} \\
& \left.\times \int_{0}^{\infty} \mathscr{R}(s) e^{\varepsilon s}\left(\int_{t_{k}+0-s}^{t_{k}+0} U_{i}(z) d z\right) d s\right], \\
& \leq \sum_{i=1}^{n} \lambda_{i}\left[\eta_{k}^{r} U_{i}(t)+\sum_{j=1}^{m} c_{i j}^{+} L_{j}^{f} \int_{0}^{\infty} \mathscr{K} e^{\varepsilon s}\left(\int_{t-s}^{t} V_{j}(z) d z\right) d s\right. \\
& +\sum_{j=1}^{m} \sum_{q=1}^{m}\left(s_{i j q}^{+}+s_{i q j}^{+}\right) M_{q}^{f} L_{j}^{f} \\
& \left.\times \int_{0}^{\infty} \mathscr{R}(s) e^{\varepsilon s}\left(\int_{t-s}^{t} V_{j}(z) d z\right) d s\right], \\
& \sum_{j=1}^{n} \lambda_{n+j}\left[\eta_{k}^{r} V_{j}(t)\right. \\
& +\sum_{i=1}^{n} d_{j i}^{+} L_{i}^{g} \int_{0}^{\infty} \mathscr{K}(s) e^{\varepsilon s}\left(\int_{t-s}^{t} U_{i}(z) d z\right) d s \\
& +\sum_{i=1}^{n} \sum_{p=1}^{n}\left(e_{j i p}^{+}+e_{j p i}^{+}\right) M_{p}^{g} L_{i}^{g} \\
& \left.\times \int_{0}^{\infty} \mathscr{R}(s) e^{\varepsilon s}\left(\int_{t-s}^{t} U_{i}(z) d z\right) d s\right] \\
& \leq \eta_{k}^{r} \mathbb{V}\left(t_{k}\right)
\end{aligned}
$$

Now, we claim that

$$
\mathbb{V}\left(t_{k}+0\right) \leq \eta_{k}^{r} \eta_{k-1}^{r} \cdots \eta_{1}^{r} \mathbb{V}\left(t_{0}\right), \quad t \in\left(t_{k-1}, t_{k}\right], k \in \mathbb{Z} .
$$

In fact, for $t \in\left(t_{0}, t_{1}\right]$, noticing that $\eta_{1} \geq 1$ and (25), we have

$$
\eta_{1}^{r} \mathbb{V}\left(t_{1}\right) \leq \eta_{1}^{r} \mathbb{V}\left(t_{0}\right)
$$

On the other hand, from (26), we have

$$
\mathbb{V}\left(t_{1}+0\right) \leq \eta_{1}^{r} \mathbb{V}\left(t_{1}\right)
$$

Combining (28) and (29), we obtain

$$
\mathbb{V}\left(t_{1}+0\right) \leq \eta_{1}^{r} \mathbb{V}\left(t_{0}\right),
$$

which implies that (27) holds for $k=1$. Assume that (27) holds for $k=m$, that is,

$$
\mathbb{V}\left(t_{m}+0\right) \leq \eta_{m}^{r} \eta_{m-1}^{r} \cdots \eta_{1}^{r} \mathbb{V}\left(t_{0}\right)
$$

Then, for $t \in\left(t_{m}, t_{m+1}\right]$, from (25), we have

$$
\mathbb{V}\left(t_{m+1}\right) \leq \mathbb{V}\left(t_{m}+0\right)
$$

On the other hand, from (26), we have

$$
\mathbb{V}\left(t_{m+1}+0\right) \leq \eta_{m+1}^{r} \mathbb{V}\left(t_{m+1}\right)
$$

From (31)-(33), we obtain

$$
\mathbb{V}\left(t_{m+1}+0\right) \leq \eta_{m+1}^{r} \eta_{m}^{r} \cdots \eta_{1}^{r} \mathbb{V}\left(t_{0}\right)
$$

This shows that (27) holds for $k=m+1$. Hence, by mathematical induction, (27) holds for all $k \in \mathbb{Z}$. Combining (25) and (27), we obtain

$$
\mathbb{V}(t) \leq \mathbb{V}\left(t_{k}+0\right) \leq \eta_{k}^{r} \eta_{k-1}^{r}, \cdots, \eta_{1}^{r} \mathbb{V}\left(t_{0}\right)
$$

for all $t \in\left(t_{k}, t_{k+1}\right], k \in \mathbb{Z}$. Noticing that $\eta_{k}^{r} \leq e^{\theta\left(t_{k}-t_{k-1}\right)}, k \in \mathbb{Z}$ in $\left(S_{6}\right)$, we have

$$
\mathbb{V}(t) \leq \mathbb{V}\left(t_{0}\right) e^{\theta\left(t_{k}-t_{k-1}\right)} e^{\theta\left(t_{k-1}-t_{k-2}\right)} \cdots e^{\theta\left(t_{1}-t_{0}\right)} \leq \mathbb{V}(0) e^{\theta t}
$$

for all $t \in\left(t_{k}, t_{k+1}\right], k \in \mathbb{Z}$. On the other hand, it follows from (21) that

$\mathbb{V}(t)$

$$
\begin{aligned}
\geq \lambda^{-} & {\left[\sum_{i=1}^{n} U_{i}(t)+\sum_{j=1}^{m} V_{j}(t)\right] } \\
=\lambda^{-} & {\left[\sum_{i=1}^{n}\left|x_{i}(t, \phi)-x_{i}(t, \varphi)\right|^{r}\right.} \\
& \left.+\sum_{j=1}^{m}\left|y_{j}(t, \phi)-y_{j}(t, \varphi)\right|^{r}\right] e^{\varepsilon t},
\end{aligned}
$$

$\mathbb{V}(0)$

$$
\begin{aligned}
=\sum_{i=1}^{n} \lambda_{i}\left[U_{i}(0)\right. & \\
& +\sum_{j=1}^{m} c_{i j}^{+} L_{j}^{f} \int_{0}^{\infty} \mathscr{K}(s) e^{\varepsilon s}\left(\int_{-s}^{0} V_{j}(z) d z\right) d s
\end{aligned}
$$




$$
\begin{aligned}
& +\sum_{j=1}^{m} \sum_{q=1}^{m}\left(s_{i j q}^{+}+s_{i q j}^{+}\right) M_{q}^{f} L_{j}^{f} \\
& \left.\times \int_{0}^{\infty} \mathscr{R}(s) e^{\varepsilon s}\left(\int_{-s}^{0} V_{j}(z) d z\right) d s\right] \\
& +\sum_{j=1}^{n} \lambda_{n+j}\left[V_{j}(0)\right. \\
& +\sum_{i=1}^{n} d_{j i}^{+} L_{i}^{g} \int_{0}^{\infty} \mathscr{K}(s) e^{\varepsilon s}\left(\int_{-s}^{0} U_{i}(z) d z\right) d s \\
& +\sum_{i=1}^{n} \sum_{p=1}^{n}\left(e_{j i p}^{+}+e_{j p i}^{+}\right) M_{p}^{g} L_{i}^{g} \\
& \left.\times \int_{0}^{\infty} \mathscr{R}(s) e^{\varepsilon s}\left(\int_{-s}^{0} U_{i}(z) d z\right) d s\right] \\
& \leq \lambda^{+} \Lambda\left\{\sum_{j=1}^{m} \sup _{s \in(-\infty, 0]}\left|\phi_{x_{i}}(s)-\psi_{x_{i}}(s)\right|^{r}\right. \\
& \left.+\sum_{i=1}^{n} \sup _{s \in(-\infty, 0]}\left|\phi_{y_{j}}(s)-\psi_{y_{j}}(s)\right|^{r}\right\},
\end{aligned}
$$

where

$$
\begin{gathered}
\lambda^{+}=\max \left\{\lambda_{1}, \lambda_{2}, \ldots, \lambda_{n+m}\right\} \\
\lambda^{-}=\min \left\{\lambda_{1}, \lambda_{2}, \ldots, \lambda_{n+m}\right\} \\
\Lambda=\max \left\{\left[1+\sum_{i=1}^{n} \max _{1 \leq j \leq m} d_{j i}^{+} L_{i}^{g} \int_{0}^{\infty} \mathscr{K}(s) e^{\varepsilon s}\left(\int_{-s}^{0} e^{\varepsilon z} d z\right) d s\right.\right. \\
+\sum_{i=1}^{n} \sum_{p=1}^{n} \max _{1 \leq j \leq m}\left(e_{j i p}^{+}+e_{j p i}^{+}\right) M_{p}^{g} L_{i}^{g} \\
\left.\times \int_{0}^{\infty} \mathscr{R}(s) e^{\varepsilon s}\left(\int_{-s}^{0} e^{\varepsilon z} d z\right) d s\right] \\
1+\sum_{j=1}^{m} \max _{1 \leq i \leq n}^{+} L_{j}^{f} \int_{0}^{\infty} \mathscr{K}(s) e^{\varepsilon s}\left(\int_{-s}^{0} e^{\varepsilon z} d z\right) d s \\
+\sum_{j=1}^{m} \sum_{q=1}^{m} \max _{1 \leq i \leq n}\left(s_{i j q}^{+}+s_{i q j}^{+}\right) M_{q}^{f} L_{j}^{f} \\
\left.\left.\times \int_{0}^{\infty} \mathscr{R}(s) e^{\varepsilon s}\left(\int_{-s}^{0} e^{\varepsilon z} d z\right) d s\right]\right\}
\end{gathered}
$$

Together with (36)-(37), we have

$$
\begin{aligned}
& \sum_{i=1}^{n}\left|x_{i}(t, \phi)-x_{i}(t, \varphi)\right|^{r}+\sum_{j=1}^{m}\left|y_{j}(t, \phi)-y_{j}(t, \varphi)\right|^{r} \\
& \leq \frac{\lambda^{+}}{\lambda^{-}} \Lambda e^{-(\varepsilon-\theta) t}\|\phi-\varphi\|_{\Delta^{\prime}}^{r}
\end{aligned}
$$

for all $t \geq 0$. Let $\mathscr{M}=\left(\left(\lambda^{+} / \lambda^{-}\right) \Lambda\right)^{1 / r}, \alpha=(\varepsilon-\theta) / r$ and then we have

$$
\|z(t, \phi)-z(t, \varphi)\|_{r} \leq \mathscr{M} e^{-\alpha t}\|\phi-\varphi\|_{\Delta} .
$$

This completes the proof.

In the following, we will study the existence, uniqueness, and global exponential stability of periodic solution of system (1) by exploiting Lemmas 5 and 6.

Theorem 7. Assume that assumptions $\left(S_{1}\right)-\left(S_{6}\right)$ hold, then system (1) has a unique $\omega$-periodic solution, which is globally exponentially stable.

Proof. Firstly, we prove the existence of periodic solution of system (1). To this end, let $z(t, \varphi)=\left(x_{1}(t, \varphi)\right.$, $\left.x_{2}(t, \varphi), \ldots, x_{n}(t, \varphi), y_{1}(t, \varphi), y_{2}(t, \varphi), \ldots, y_{m}(t, \varphi)\right)^{T}$ be an arbitrary solution of system (1) through $(0, \varphi)$, where $\varphi \in C^{*}$. Define $z(t, \phi)=z(t+\omega, \varphi)$, where $\phi=z(s+\omega, \varphi), s \leq 0$. We can know that $\phi \in C^{*}$ and $z(t, \phi)=$ $\left(x_{1}(t, \phi), x_{2}(t, \phi), \ldots, x_{n}(t, \phi), y_{1}(t, \phi), y_{2}(t, \phi), \ldots, y_{m}(t, \phi)\right)^{T}$ is also a solution of system (1) through $(0, \phi)$. By virtue of Lemma 6, we have

$$
\begin{aligned}
& \left\{\sum_{i=1}^{n}\left|x_{i}(t, \phi)-x_{i}(t, \varphi)\right|^{r}+\sum_{j=1}^{m}\left|y_{i}(t, \phi)-y_{i}(t, \varphi)\right|^{r}\right\}^{1 / r} \\
& \quad \leq M e^{-\alpha t}\|\phi-\varphi\|_{\Delta},
\end{aligned}
$$

for $t \geq 0$. So, we have

$$
\begin{aligned}
& \left\{\sum_{i=1}^{n}\left|x_{i}(t+\omega, \varphi)-x_{i}(t, \varphi)\right|^{r}\right. \\
& \left.\quad+\sum_{j=1}^{m}\left|y_{j}(t+\omega, \varphi)-y_{j}(t, \varphi)\right|^{r}\right\}^{1 / r} \\
& \leq \mathscr{M} e^{-\alpha t}\|\phi-\varphi\|_{\Delta},
\end{aligned}
$$

for $t \geq 0$. It follows from Lemma 5 that

$$
\begin{aligned}
& \sum_{i=1}^{n}\left|x_{i}(t+\omega, \varphi)-x_{i}(t, \varphi)\right|+\sum_{j=1}^{m}\left|y_{j}(t+\omega, \varphi)-y_{j}(t, \varphi)\right| \\
& \leq(n+m)^{1-1 / r} \mathscr{M} e^{-\alpha t}\|\phi-\varphi\|_{\Delta} .
\end{aligned}
$$


Noticing that for $i=1,2, \ldots, n$,

$$
\begin{aligned}
& x_{i}(t+k \omega, \varphi) \\
& \quad=x_{i}(t, \varphi)+\sum_{s=1}^{k}\left[x_{i}(t+s \omega, \varphi)-x_{i}(t+(s-1) \omega, \varphi)\right] .
\end{aligned}
$$

It follows from (43)-(44) that

$$
\begin{aligned}
& \lim _{k \rightarrow \infty} x_{i}(t+k \omega, \varphi) \\
& \quad x_{i}(t, \varphi)+\lim _{k \rightarrow \infty} \sum_{s=1}^{k}\left[x_{i}(t+s \omega, \varphi)-x_{i}(t+(s-1) \omega, \varphi)\right] \\
& \quad \leq x_{i}(t, \varphi)+(n+m)^{(1-1 / r)} \mathscr{M}\|\phi-\varphi\|_{\Delta} \lim _{k \rightarrow \infty} \sum_{s=1}^{k} e^{-\alpha(t+(s-1) \omega)} \\
& \quad \leq x_{i}(t, \varphi)+(n+m)^{(1-1 / r)} \mathscr{M} e^{-\alpha t}\|\phi-\varphi\|_{\Delta} \sum_{s=1}^{\infty} e^{-\alpha(s-1) \omega} \\
& \quad<\infty,
\end{aligned}
$$

which implies that $\lim _{k \rightarrow \infty} x_{i}(t+k \omega, \varphi)$ exists. Similar to (44) and (45), we obtain that $\lim _{k \rightarrow \infty} y_{j}(t+k \omega, \varphi)$ exists. Let

$$
\begin{aligned}
z^{*}\left(t, \varphi^{*}\right) & \\
= & \left(x_{1}^{*}\left(t, \varphi^{*}\right), x_{2}^{*}\left(t, \varphi^{*}\right), \ldots, x_{n}^{*}\left(t, \varphi^{*}\right),\right. \\
& \left.y_{1}^{*}\left(t, \varphi^{*}\right), y_{2}^{*}\left(t, \varphi^{*}\right), \ldots, y_{m}^{*}\left(t, \varphi^{*}\right)\right)^{T},
\end{aligned}
$$

where $\lim _{k \rightarrow \infty} x_{i}(t+k \omega, \varphi)=x_{i}^{*}\left(t, \varphi^{*}\right), \lim _{k \rightarrow \infty} y_{j}(t+$ $k \omega, \varphi)=y_{j}^{*}\left(t, \varphi^{*}\right)$ for $i=1,2, \ldots, n, j=1,2, \ldots, m$. Then $z^{*}\left(t, \varphi^{*}\right)$ is an $\omega$-periodic solution for system (1).

Secondly, we prove the uniqueness of periodic solution of system (1). Assume that $z(t, \zeta)=\left(x_{1}(t, \zeta)\right.$, $\left.x_{2}(t, \zeta), \ldots, x_{n}(t, \zeta), y_{1}(t, \zeta), y_{2}(t, \zeta), \ldots, y_{m}(t, \zeta)\right)^{T}$ is another $\omega$-periodic solution of system (1) through $(0, \zeta)$, where $\zeta \in$ $C^{*}$. By a minor modification of the proof of $(43)$, we have

$$
\begin{aligned}
& \sum_{i=1}^{n}\left|x_{i}^{*}\left(t, \varphi^{*}\right)-x_{i}(t, \zeta)\right|+\sum_{j=1}^{m}\left|y_{j}^{*}\left(t, \varphi^{*}\right)-y_{j}(t, \zeta)\right| \\
& =\sum_{i=1}^{n}\left|x_{i}^{*}\left(t+k \omega, \varphi^{*}\right)-x_{i}(t, \zeta)\right| \\
& \quad+\sum_{j=1}^{m}\left|y_{j}^{*}\left(t+k \omega, \varphi^{*}\right)-y_{j}(t, \zeta)\right| \\
& \leq(n+m)^{1-1 / r} \mathscr{M} e^{-\alpha(t+k \omega)}\left\|\varphi^{*}-\zeta\right\|_{\Delta^{\prime}}, \quad t \geq 0 .
\end{aligned}
$$

Taking $k \rightarrow \infty$, we have

$$
\begin{aligned}
& x_{i}^{*}\left(t, \varphi^{*}\right)=x_{i}(t, \zeta), \quad t \geq 0, i=1,2, \ldots, n, \\
& y_{j}^{*}\left(t, \varphi^{*}\right)=y_{j}(t, \zeta), \quad t \geq 0, j=1,2, \ldots, m,
\end{aligned}
$$

which implies that system (1) has a unique $\omega$-periodic solution.

Finally, since $z^{*}\left(t, \varphi^{*}\right)$ is a unique $\omega$-periodic solution of system (1), let $z(t, \psi)$ be any other solution of system (1) through $(0, \psi)$. From Lemma 6 , we obtained that

$$
\left\|z(t, \psi)-z^{*}\left(t, \varphi^{*}\right)\right\|_{r} \leq M e^{-\alpha t}\left\|\psi-\varphi^{*}\right\|_{\Delta}, \quad t \geq 0,
$$

where $\psi, \varphi^{*} \in C^{*}$ and $\alpha, \mathscr{M}$ are the same as defined in Lemma 6. It follows from Definition 2 that the $\omega$-periodic solution $z^{*}\left(t, \varphi^{*}\right)$ is globally exponentially stable. Up to now, we conclude that system (1) has a unique $\omega$-periodic solution $z^{*}\left(t, \varphi^{*}\right)$, which is globally exponentially stable. This completes the proof.

Remark 8. In assumption $\left(\mathrm{S}_{5}\right)$, we only assume that the impulsive operators $H_{i k}(\cdot)$ and $E_{j k}(\cdot)$ are Lipschitz continuous, which remove the usual assumptions that the boundedness and linearity of the impulsive operators are required in $[18,19,21,29-31]$. Thus, our results have wider adaptive range. Particularly, if we take the linear operators $\Delta x_{i}\left(t_{k}\right)=$ $-\gamma_{i k} x_{i}\left(t_{k}^{-}\right)$and $\Delta y_{j}\left(t_{k}\right)=-\alpha_{j k} y_{j}\left(t_{k}^{-}\right)$as considered in $[18,19$, $21,29-31]$, that is,

$$
\left(\mathrm{S}_{7}\right)\left\{\begin{array}{cc}
H_{i k}\left(x_{i}\left(t_{k}^{-}\right)\right)=\left(1-\gamma_{i k}\right) x_{i}\left(t_{k}^{-}\right), & \gamma_{i k} \in(0,2), i=1,2, \ldots, n, k=1,2, \ldots, \\
E_{j k}\left(y_{j}\left(t_{k}^{-}\right)\right)=\left(1-\alpha_{j k}\right) y_{j}\left(t_{k}^{-}\right), & \alpha_{j k} \in(0,2), j=1,2, \ldots m, k=1,2, \ldots,
\end{array}\right.
$$

then we have $H_{i k}=\left|1-\gamma_{i k}\right|<1, E_{j k}=\left|1-\alpha_{j k}\right|<1$. So we can choose $\eta_{k}=1$ and $\theta=0$ to satisfy assumption $\left(\mathrm{S}_{6}\right)$. In this case, we have the following interesting corollary.

Corollary 9. Assume that assumptions $\left(\left(S_{1}\right)-\left(S_{4}\right)\right)$ and $\left(S_{7}\right)$ hold; then system (1) has a unique $\omega$-periodic solution, which is globally exponentially stable.

Remark 10. Note that when $t_{k}-t_{k-1}=\infty, k=1,2, \ldots$ in assumption $\left(\mathrm{S}_{6}\right)$, which implies that there are no impulsive effects on system (1). Correspondingly, we call system (1) an impulse-free. In this case, we have the following corollary.

Corollary 11. Assume that assumptions $\left(\left(S_{1}\right)-\left(S_{4}\right)\right)$ hold; then the impulse-free system (1) has a unique $\omega$-periodic solution, which is globally exponentially stable.

Remark 12. Clearly, based on the general $\|\cdot\|_{r}$ and Lemma 5, a general criterion ensuring the existence of periodic solution and its global exponential stability of system (1) with and without impulses has been established. Compared with results in $[6,7,15-17]$, it is easy to see that our results are extended and improved because their results can be viewed as the special case of $r=1$ in assumption $\left(\mathrm{S}_{4}\right)$. In addition, since the nonnetwork parameter $r$ is introduced in the condition $\left(\mathrm{S}_{4}\right)$, it can allow much broader applications for designing the circuit of a convergent impulsive network.

Remark 13. In assumption $\left(\mathrm{S}_{3}\right)$, if the kernel is a delta function of the form:

$$
\begin{aligned}
K_{i j}(s) & =R_{i j q}(s)=\delta(s-\tau), \\
\widetilde{K}_{j i}(s) & =\widetilde{R}_{j i p}(s)=\delta(s-\sigma),
\end{aligned}
$$


where $\tau \geq 0$ and $\sigma \geq 0$, then system (1) with continuously distributed delays reduces to the model with discrete delays in [29]. According to Lemma 3, we know that the condition $\left(\mathrm{H}_{5}\right)$ of Theorem 3.1 in [29] implies that $\Omega$ with $r=1$ is an $M$-matrix but not vice versa. Thus, our results are new and complementary to their results.

\section{An Example}

In this section, an example and its numerical simulation are given to illustrate the correctness of the obtained theoretical results.

An Example. Consider the following high-order BAM neural networks with infinite distributed delays and impulses:

$$
\begin{aligned}
& \frac{d x_{1}(t)}{d t} \\
& =-(8+\sin 2 t) x_{1}(t) \\
& +(2+\cos 2 t) \int_{0}^{\infty} e^{-s} f_{1}\left(y_{1}(t-s)\right) d s \\
& +2 \sin 2 t \int_{0}^{\infty} s e^{-s} f_{1}\left(y_{1}(t-s)\right) f_{1}\left(y_{1}(t-s)\right) d s \\
& +\cos 2 t, \quad t \neq t_{k}, \\
& x_{1}\left(t_{k}^{+}\right) \\
& =x_{1}\left(t_{k}^{-}\right)+\Delta x_{1}\left(t_{k}\right)=H_{1 k}\left(x_{1}\left(t_{k}^{-}\right)\right), \quad k=1,2, \ldots, \\
& \frac{d y_{1}(t)}{d t} \\
& =-(10-3 \cos 2 t) y_{1}(t) \\
& +(1+\sin 2 t) \int_{0}^{\infty} e^{-s} g_{1}\left(x_{1}(t-s)\right) d s \\
& +2 \cos 2 t \int_{0}^{\infty} s e^{-s} g_{1}\left(x_{1}(t-s)\right) g_{1}\left(x_{1}(t-s)\right) d s \\
& +\sin 2 t, \quad t \neq t_{k}, \\
& y_{1}\left(t_{k}^{+}\right) \\
& =y_{1}\left(t_{k}^{-}\right)+\Delta y_{1}\left(t_{k}\right)=E_{1 k}\left(y_{1}\left(t_{k}^{-}\right)\right), \quad k=1,2, \ldots,
\end{aligned}
$$

where $f_{1}(u)=g_{1}(u)=\tanh (u)$. By simple calculation, we obtain that $L_{1}^{g}=L_{1}^{f}=M_{1}^{f}=M_{1}^{g}=1$ and

$$
\Sigma=\left(\begin{array}{cc}
7 & -7 \\
-6 & 6+r
\end{array}\right) \text {. }
$$

If the integer $r \geq 1$, then $\Sigma$ is an $M$-matrix. Thus, assumptions $\left(\left(S_{1}\right)-\left(S_{4}\right)\right)$ are satisfied for system (51). For the impulsive part, the following two cases are considered.

Case 1. When $t_{k}-t_{k-1}=\infty, k=1,2, \ldots$, by Corollary 11, we conclude that the impulse-free system (51) has a unique $\pi$-periodic solution, which is globally exponentially stable.
Case 2. When the impulsive parts are taken as the nonlinear operators such that $\Delta x_{1}\left(t_{k}\right)=\gamma \tan \left(x_{1}\left(t_{k}^{-}\right)\right), \gamma \in(0,2)$ and $\Delta y_{1}\left(t_{k}\right)=\gamma \tan \left(y_{1}\left(t_{k}^{-}\right)\right), \gamma \in(0,2)$, that is,

$$
\begin{array}{r}
H_{1 k}\left(x_{i}\left(t_{k}^{-}\right)\right)=x_{1}\left(t_{k}^{-}\right)-\gamma \tan \left(x_{1}\left(t_{k}^{-}\right)\right), \\
\gamma \in(0,2), k=1,2, \ldots, \\
E_{1 k}\left(y_{j}\left(t_{k}^{-}\right)\right)=y_{1}\left(t_{k}^{-}\right)-\gamma \tan \left(y_{1}\left(t_{k}^{-}\right)\right), \\
\gamma \in(0,2), \quad k=1,2, \ldots,
\end{array}
$$

which satisfy the assumption $\left(\mathrm{S}_{5}\right)$ with Lipschitz constants $H_{1 k}=E_{1 k} \leq|1-\gamma|<1, k=1,2, \ldots$. So we can choose $\eta_{k}=1$ and $\theta=0$ to satisfy the assumption $\left(\mathrm{S}_{6}\right)$. According to Theorem 7, we conclude that the impulsive system (51) has a unique $\pi$-periodic solution, which is globally exponentially stable. However, in this case, results in [18, 19, 21, 29-31] are ineffective because the function $\tan (\cdot)$ is nonlinear. Moreover, the condition $\left(\mathrm{H}_{5}\right)$ of Theorem 3.1 in [29] cannot be applied to system (51), since $b_{1}^{-}-c_{11}^{+} L_{1}^{f}-2 s_{111}^{+} M_{1}^{f} L_{1}^{f}=0 \ngtr 0$.

\section{Conclusions}

In this paper, we have studied the existence, uniqueness, and global exponential stability of periodic solution for a kind of high-order BAM neural networks with continuously distributed delays and general impulses. It should be noted that some extended and improved criteria have been derived by exploiting the general $\|\cdot\|_{r}$, Lemma 5 , and the Lyapunov functional method. In addition, these criteria are in terms of $M$-matrix, which can be easily checked by many equivalent conditions listed in [32]. Finally, an example and its numerical simulation are given to show the feasibility and correctness of the obtained results.

\section{Acknowledgments}

The authors appreciate the anonymous reviewer for her/his insightful comments and valuable suggestions, which have led to a great improvement in this paper. This research is supported by the Chinese Universities Specialized Research Fund for the Doctoral Program (20110185110020), the Research Fund of Kunming University (XJ11L019, XJL11008), and the Fundamental Research Funds for the Central Universities (ZYGX2010J106).

\section{References}

[1] P. Baldi and A. F. Atiya, "How delays affect neural dynamics and learning," IEEE Transactions on Neural Networks, vol. 5, no. 4, pp. 612-621, 1994.

[2] J. Bélair, S. A. Campbell, and P. Van Den Driessche, "Frustration, stability, and delay-induced oscillations in a neural network model," SIAM Journal on Applied Mathematics, vol. 56, no. 1, pp. 245-255, 1996.

[3] X. Liu, "Stability results for impulsive differential systems with applications to population growth models," Dynamics \& Stability of Systems, vol. 9, no. 2, pp. 163-174, 1994. 
[4] H. Ye, A. N. Michel, and L. Hou, "Stability analysis of systems with impulse effects," IEEE Transactions on Automatic Control, vol. 43, no. 12, pp. 1719-1723, 1998.

[5] K. Gopalsamy and X. Z. He, "Delay-independent stability in bidirectional associative memory networks," IEEE Transactions on Neural Networks, vol. 5, no. 6, pp. 998-1002, 1994.

[6] J. Cao and L. Wang, "Exponential stability and periodic oscillatory solution in BAM networks with delays," IEEE Transactions on Neural Networks, vol. 13, no. 2, pp. 457-463, 2002.

[7] H. Huang and J. Cao, "On global asymptotic stability of recurrent neural networks with time-varying delays," Applied Mathematics and Computation, vol. 142, no. 1, pp. 143-154, 2003.

[8] X. Yang, C. Li, X. Liao, D. J. Evans, and G. M. Megson, "Global exponential periodicity of a class of bidirectional associative memory networks with finite distributed delays," Applied Mathematics and Computation, vol. 171, no. 1, pp. 108-121, 2005.

[9] T. Zhou, A. Chen, and Y. Zhou, "Existence and global exponential stability of periodic solution to BAM neural networks with periodic coefficients and continuously distributed delays," Physics Letters A, vol. 343, no. 5, pp. 336-350, 2005.

[10] J. Shao, T. Huang, and X. Wang, "Improved global robust exponential stability criteria for interval neural networks with time-varying delays," Expert Systems With Applications, vol. 38, pp. 15587-15593, 2011.

[11] S. L. Wu, K. L. Li, and T. Z. Huang, "Exponential stability of static neural networks with time delay and impulses," IET Control Theory and Applications, vol. 5, no. 8, pp. 943-951, 2011.

[12] I. M. Stamova, R. Ilarionov, and R. Vaneva, "Impulsive control for a class of neural networks with bounded and unbounded delays," Applied Mathematics and Computation, vol. 216, no. 1, pp. 285-290, 2010.

[13] J. J. Hopfield and D. W. Tank, "Computing with neural circuits A," Science, vol. 233, no. 4764, pp. 625-633, 1986.

[14] B. Kosko, "Bi-dierctional associative memories," IEEE Transactions on Systems, Man and Cybernetics, vol. 18, no. 1, pp. 49-60, 1988.

[15] Y. T. Li and C. B. Yang, "Global exponential stability analysis on impulsive BAM neural networks with distributed delays," Journal of Mathematical Analysis and Applications, vol. 324, no. 2, pp. 1125-1139, 2006.

[16] Y. Xia, Z. Huang, and M. Han, "Existence and globally exponential stability of equilibrium for BAM neural networks with impulses," Chaos, Solitons and Fractals, vol. 37, no. 2, pp. 588597, 2008.

[17] K. Li, "Delay-dependent stability analysis for impulsive BAM neural networks with time-varying delays," Computers and Mathematics with Applications, vol. 56, no. 8, pp. 2088-2099, 2008.

[18] Y. T. Li and J. Wang, "An analysis on the global exponential stability and the existence of periodic solutions for nonautonomous hybrid BAM neural networks with distributed delays and impulses," Computers and Mathematics with Applications, vol. 56, no. 9, pp. 2256-2267, 2008.

[19] Z. Gui, X. S. Yang, and W. Ge, "Existence and global exponential stability of periodic solutions of recurrent cellular neural networks with impulses and delays," Mathematics and Computers in Simulation, vol. 79, no. 1, pp. 14-29, 2008.

[20] H. Gu, H. Jiang, and Z. Teng, "BAM-type impulsive neural networks with time-varying delays," Nonlinear Analysis: Real World Applications, vol. 10, no. 5, pp. 3059-3072, 2009.
[21] Y. Shao and B. Dai, "The existence of exponential periodic attractor of impulsive BAM neural network with periodic coefficients and distributed delays," Neurocomputing, vol. 73, no. 16-18, pp. 3123-3131, 2010.

[22] X. Li, "Existence and global exponential stability of periodic solution for impulsive Cohen-Grossberg-type BAM neural networks with continuously distributed delays," Applied Mathematics and Computation, vol. 215, no. 1, pp. 292-307, 2009.

[23] E. B. Kosmatopoulos, M. M. Polycarpou, M. A. Christodoulou, and P. A. Ioannou, "High-order neural network structures for identification of dynamical systems," IEEE Transactions on Neural Networks, vol. 6, no. 2, pp. 422-431, 1995.

[24] P. K. Simpson, "Higher-ordered and intraconnected bidirectional associative memories," IEEE Transactions on Systems, Man and Cybernetics, vol. 20, no. 3, pp. 637-653, 1990.

[25] D. W. C. Ho, J. Liang, and J. Lam, "Global exponential stability of impulsive high-order BAM neural networks with time-varying delays," Neural Networks, vol. 19, no. 10, pp. 1581-1590, 2006.

[26] H. Jiang and J. Liu, "Danamics analysis of impulsive stochastic high-order BAM neu ral networks with markovian jumping and mixed delays," International Journal of Computer Mathematics, vol. 4, pp. 149-170, 2011.

[27] H. Gu, "Mean square exponential stability in high-order stochastic impulsive BAM neural networks with time-varying delays," Neurocomputing, vol. 74, no. 5, pp. 720-729, 2011.

[28] C. Li, C. Li, X. Liao, and T. Huang, "Impulsive effects on stability of high-order BAM neural networks with time delays," Neurocomputing, vol. 74, no. 10, pp. 1541-1550, 2011.

[29] H. F. Huo, W. T. Li, and S. Tang, "Dynamics of high-order BAM neural networks with and without impulses," Applied Mathematics and Computation, vol. 215, no. 6, pp. 2120-2133, 2009.

[30] W. Yang, "Existence and stability of periodic solutions of BAM high-order Hopfield neural networks with impulses and delays on time scales," Electronic Journal of Differential Equations, vol. 2012, pp. 1-22, 2012.

[31] Y. Zhang and Q. G. Wang, "Stationary oscillation for highorder Hopfield neural networks with time delays and impulses," Journal of Computational and Applied Mathematics, vol. 231, no. 1, pp. 473-477, 2009.

[32] R. A. Horn and C. R. Johnson, Matrix Analysis, Cambridge University Press, Cambridge, UK, 1985. 


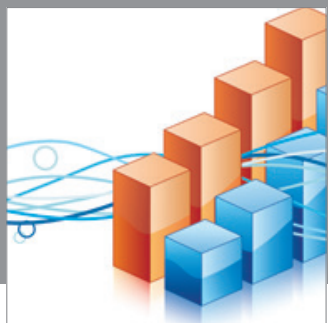

Advances in

Operations Research

mansans

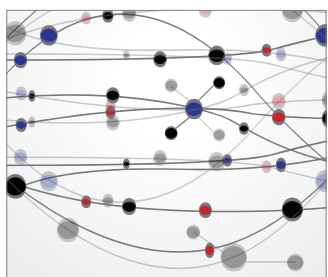

The Scientific World Journal
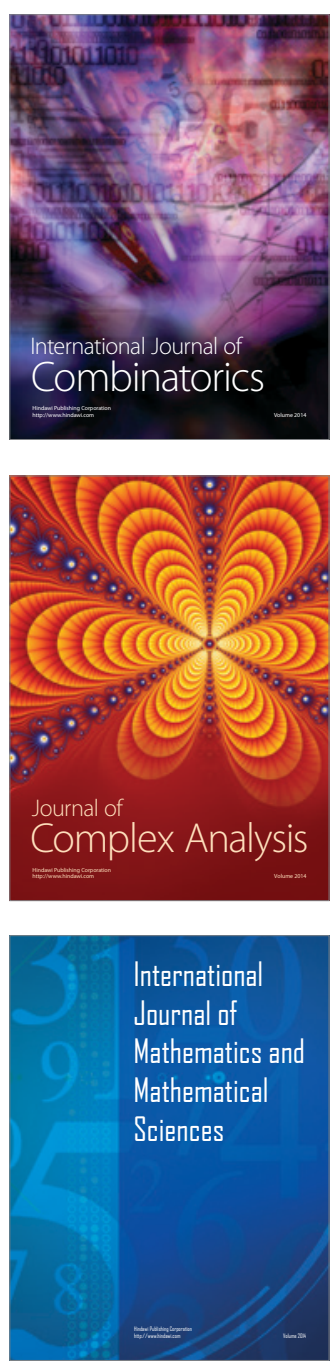
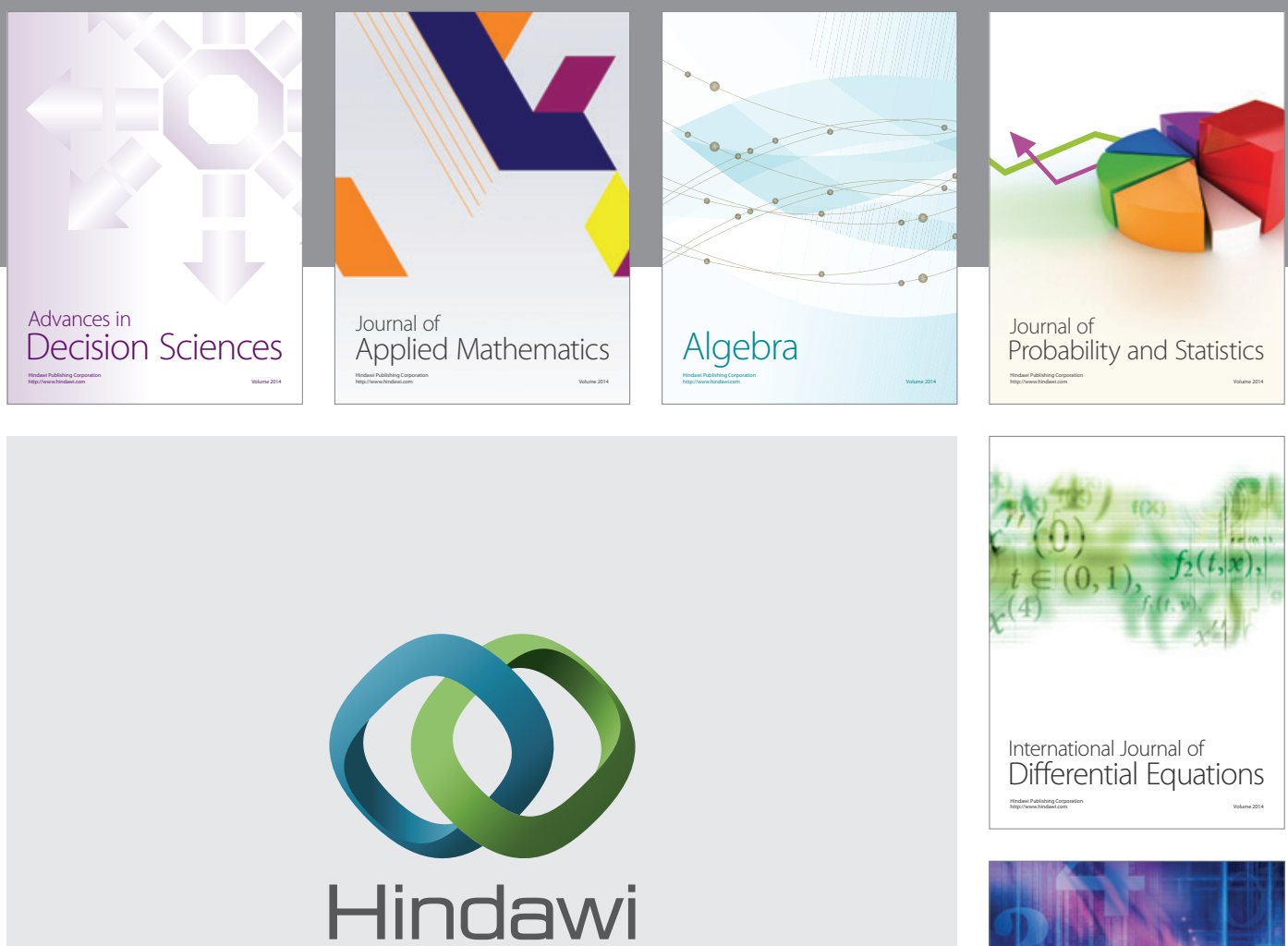

Submit your manuscripts at http://www.hindawi.com
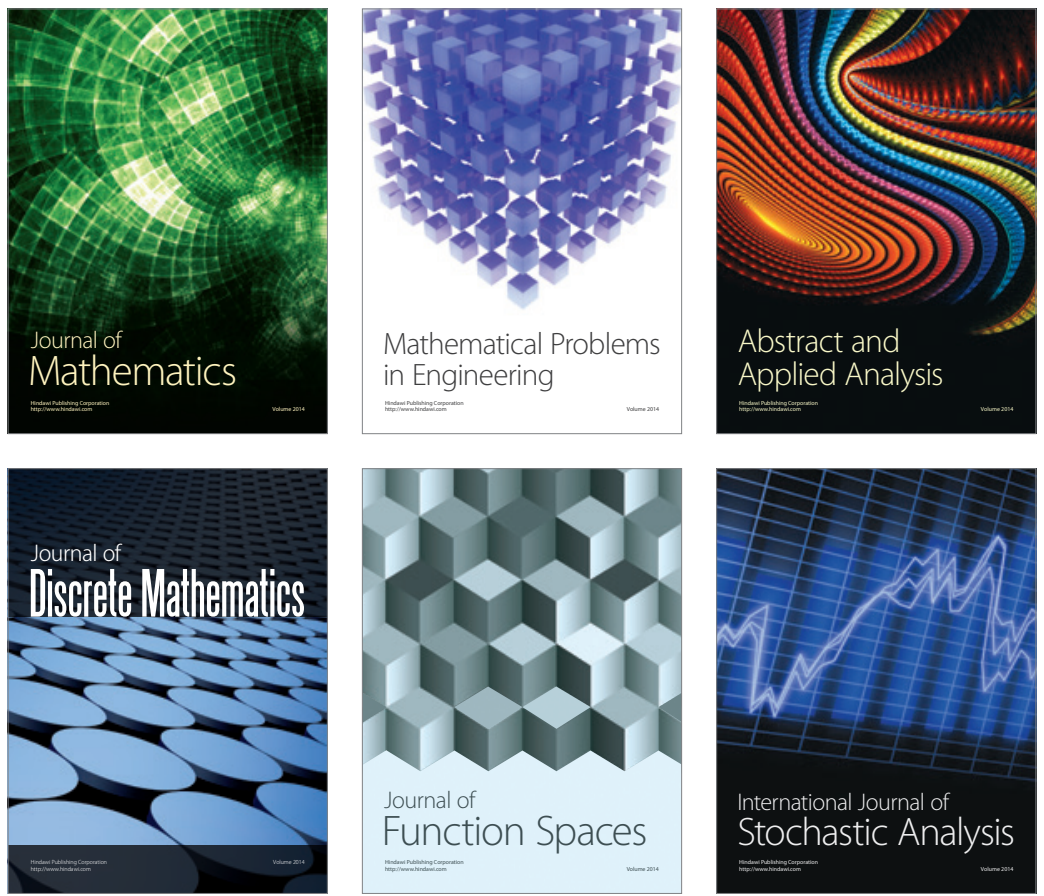

Journal of

Function Spaces

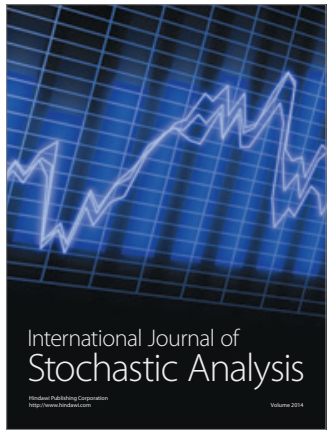

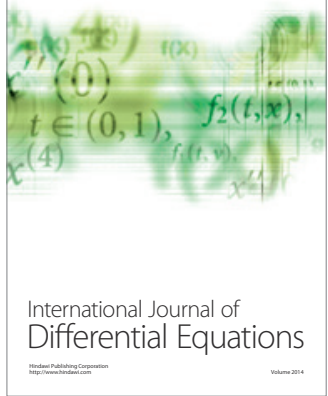
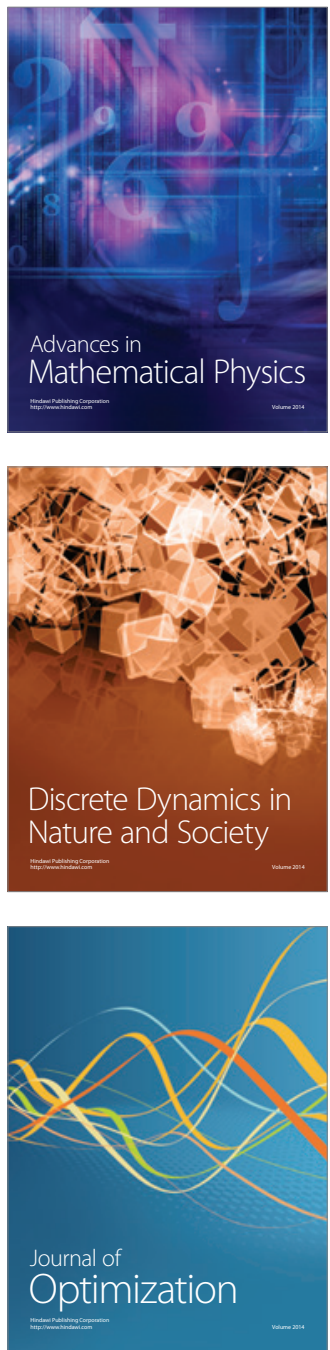